\title{
Recent Advances on Near-Infrared-Emitting Poly[2-methoxy-5-(2-ethylhexyloxy)-1,4-phenylenevinylene] Polymer Dots for in vivo Imaging
}

\author{
Liqin Xiong, ${ }^{*, a, b}$ \\ ${ }^{a}$ Nano Biomedical Research Center, School of Biomedical Engineering, Med-X Research Institute, \\ Shanghai Jiao Tong University, 1954 Huashan Road, Shanghai 200030, China \\ ${ }^{b}$ Department of Nuclear Medicine, Rui Jin Hospital, School of Medicine, Shanghai Jiao Tong University, \\ Shanghai 200025, China
}

\begin{abstract}
Conjugated polymer dots emerge as attractive molecular imaging nanoprobes in living animals for their excellent optical properties including bright fluorescence intensity, excellent photostability, high emission rates, and low intrinsic cytotoxicity. This mini-review summarizes recent advances on near-infrared-emitting poly[2-methoxy-5(2-ethylhexyloxy)-1,4-phenylenevinylene] polymer dots for in vivo bioimaging. The preparation of near-infrared MEH-PPV polymer dots is firstly discussed, followed by some examples of their applications ranging from lymph node mapping and tumor imaging to long-term tumor tracking.
\end{abstract}

Keywords near-infrared fluorescence imaging, bioluminescence, polymer dots, lymph node mapping, tumor tracking

\section{Introduction}

Fluorescence imaging has become a powerful technique to visualize biology in its native physiological settings in a living subject, ${ }^{[1-4]}$ and has been used in clinics for image-guided surgery in cancer patients. ${ }^{[5,6]}$ Besides quantum dots, metal nanoclusters, carbon nanomaterials, up-conversion nanophosphors, and silicon nanomaterials, conjugated polymer dots are promising nanoprobes and contributed to advances on fluorescence imaging. ${ }^{[7-18]}$ Conjugated polymer dots possess $\pi$-electron delocalized backbones, and their formation of spherical nanoparticles results from collapse of hydrophobic polymer chains owing to substantial decrease in solvent hydrophobicity upon going from organic solvents to water. In addition to their large absorption coefficients, bright fluorescence intensity, excellent photostability, and high emission rates, the completely organic and biologically inert components of polymer dots represent another advantage, which circumvents intrinsically the issue on heavy metal ion-induced toxicity to living organisms and thus possesses potentially good biocompatibility.

The preparation methods, optical properties, and bioconjugation chemistry of polymer dots were widely researched, and their biological and biomedical applica- tions were also demonstrated for in vitro sensing, labeling, imaging, and drug delivery in recent reviews. ${ }^{[19-32]}$ As a well-researched conjugated polymer, poly[2methoxy-5-(2-ethylhexyloxy)-1,4-phenylenevinylene] (MEH-PPV), has been extensively used in organic light-emitting diodes, solar cells, biological sensors, and imaging probes. ${ }^{[33-40]}$ However, only a few researches on the whole-body imaging of polymer dots in living subjects were reported, mainly due to the challenge on the design of polymer dots that show strong and narrow-band emissions in the near-infrared (NIR) region, and their unfavorable in vivo performance after systemic injection. ${ }^{[41-58]}$ The application of NIR fluorescence in biology enormously enhances the sensitivity of in vivo measurements and optical imaging because monitoring NIR fluorescence minimizes interference from autofluorescence in biological tissues. Herein, we will focus on recent progress in NIR fluorescence imaging based on MEH-PPV polymer dots as imaging nanoprobes. ${ }^{[59-62]}$ We will firstly discuss the design and preparation of NIR-emitting MEH-PPV polymer dots, and then highlight in vivo imaging applications of the polymer dots including lymph node mapping, tumor vascular imaging, tumor-targeted imaging, and tumor tracking. The self-luminescing NIR emission based on MEH-PPV polymer dots is also discussed.

\footnotetext{
* E-mail: xiongliqin@sjtu.edu.cn

Received October 28, 2016; accepted November 7, 2016.
} 


\section{Preparation of NIR-emitting MEH-PPV polymer dots}

Polymer dots are mainly prepared by miniemulsion and reprecipitation methods. Chiu's lab ${ }^{[18]}$ described a versatile and simple method for tuning fluorescence properties of poly(9,9-dioctylfluorene-co-benzothiadiazole) (PFBT) polymer dots by doping hydrophobic matrix of PFBT with hydrophobic dyes using a nanoscale precipitation method. This strategy offers several advantages: (1) The hydrophobic matrix of polymer dots disperses effectively and stabilizes doped dyes and allows the dyes to be used in an aqueous environment; (2) The functional groups presenting on the surface of polymer dots allow dye-doped nanoparticles to be labeled with a wide range of biomolecules; (3) Polymer dots act extremely as efficient light harvesters that greatly enhance brightness of doped dyes; (4) Polymer dots offer amplified energy transfer to the dopant, thus enabling efficient tuning of emission properties of polymer dots using the dopant. Using this strategy, NIR-emitting MEH-PPV polymer dots were prepared by encapsulating NIR dyes and silicon 2,3-naphthalocyanine bis(trihexylsilyloxide) (NIR775) into the matrix of MEH-PPV polymer dots (Scheme 1). ${ }^{[59-62]}$

The NIR fluorescence emission is produced based on a fluorescence resonance energy transfer (FRET) system utilizing NIR775 as an acceptor and MEH-PPV polymers as a donor. MEH-PPV is constructed utilizing numerous fluorescent residues that form a large conjugated system and also exhibit very fast intra- and interchain photoexcitation transport (exciton diffusion). The structure allows excitons to move along the polymer chain until they encounter a quencher molecule. This migration of excitons facilitates FRET from the MEH-PPV polymer to the NIR775 dye over even relatively long distances. The amplified FRET process is further favored by the large extinction coefficient of the NIR775 $\left(5.2 \times 10^{5} \mathrm{M}^{-1} \mathrm{~cm}^{-1}\right)$. Additionally, the hydrophobicity of NIR775 and the polymer dot matrix ensures close interaction between acceptor dyes and donor matrix, which also enhances efficient FRET. Therefore, the highly efficient FRET was observed despite poor spectral overlap between MHE-PPV's fluorescence and NIR775's absorbance. Furthermore, FRET resulted in a large Stokes shift $(>300 \mathrm{~nm})$ between excitation and emission maxima, and thus can reduce effectively high fluorescence background during in vivo imaging. Under excitation at $468 \mathrm{~nm}$, the NIR polymer dots exhibited distinctive MEH-PPV emission at $595 \mathrm{~nm}$ with a shoulder at $644 \mathrm{~nm}$, and a strong and narrow-band NIR peak at $778 \mathrm{~nm}$ with a full width at half maximum (FWHM) as narrow as $20 \mathrm{~nm}$.

The self-luminescing NIR emission is produced based on an energy transfer relay that integrates bioluminescence resonance energy transfer (BRET) and fluorescence resonance energy transfer (FRET). The good overlap between MEH-PPV absorption and Luc8 (an eight-mutation variant of $R$. reniformis luciferase) emission suggests that efficient BRET can occur. Following BRET from Luc8 to the polymer matrix, FRET could occur between the polymer matrix and the doped NIR fluorophore. This BRET-FRET relay process produces self-luminescing NIR polymer dots, enabling in vivo near-infrared imaging without external light excitation. Upon addition of coelenterazine, in addition to the emission of Luc8 at $480 \mathrm{~nm}$, two new emission peaks at $594 \mathrm{~nm}$ (relatively weak, from MEH-PPV matrix) and $778 \mathrm{~nm}$ (strongest, from NIR775) were detected.

\section{Imaging of lymphatic basins}

The lymphatic system plays a crucial role in immune response to foreign antigens and tumors, and in tumor metastasis in human and rodent models. The NIR polymer dots PD-1, PD-2, and PD-3 were applied to map lymph nodes in vivo.

The NIR polymer dots PD-2 $(\sim 10 \mu \mathrm{g})$ were introduced into the forepaws of mice via intradermal injections, the axillary lymph node (AX) could be readily visualized noninvasively by NIR fluorescence (Figure 1A). Compared with PSAM modified PD-2, PS-PEG-COOH modified PD-1 showed faster AX detection even at lower concentration $(\sim 1 \mu \mathrm{g})$ (Figure 1B).

Similarly, self-luminescing NIR polymer dots PD-3 $(\sim 2 \mu \mathrm{g})$ were introduced into the forepaws of mice via intradermal injections. Within 10 min of injection, AX could be readily visualized non-invasively by bioluminescence imaging (Figure 1C). Followed by in vivo fluorescence imaging, lower fluorescent signals were obtained at $\mathrm{AX}$ in comparison with bioluminescence imaging (Figure 1D). After PD-3 was injected into all four paws (each at $\sim 2 \mu \mathrm{g}$ ), all $\mathrm{AX}$ in the same mouse were clearly visualized by bioluminescence imaging (Figure 1E).

Furthermore, the PD-1 allows highly efficient labelling of all lymph nodes in the lymphatic networks after a single tail-vein injection. At $24 \mathrm{~h}$ after injection of the PD-1 into nude mice, the mice were imaged before and after removed superficial skin. Strong NIR fluorescence signals were detected in the lymphatic networks: neck lymph nodes, AX, lateral thoracic lymph nodes, and inguinal lymph nodes (IN) (Figure 2). ${ }^{[60]}$

\section{Imaging of tumor vasculature}

Smaller nanoprobes are often more desirable due to their favorable biodistribution characteristics in in vivo experiments. The NIR polymer dots with ultra-small size (sub-5 $\mathrm{nm}$ in diameter) were prepared for in vivo imaging. ${ }^{[61]}$

The TEM image showed that the NIR polymer dots were quite monodispersed with an average diameter of $3.6 \pm 0.4 \mathrm{~nm}$ (Figure 3A). However, DLS data showed an average hydrodynamic diameter of the NIR polymer 
A
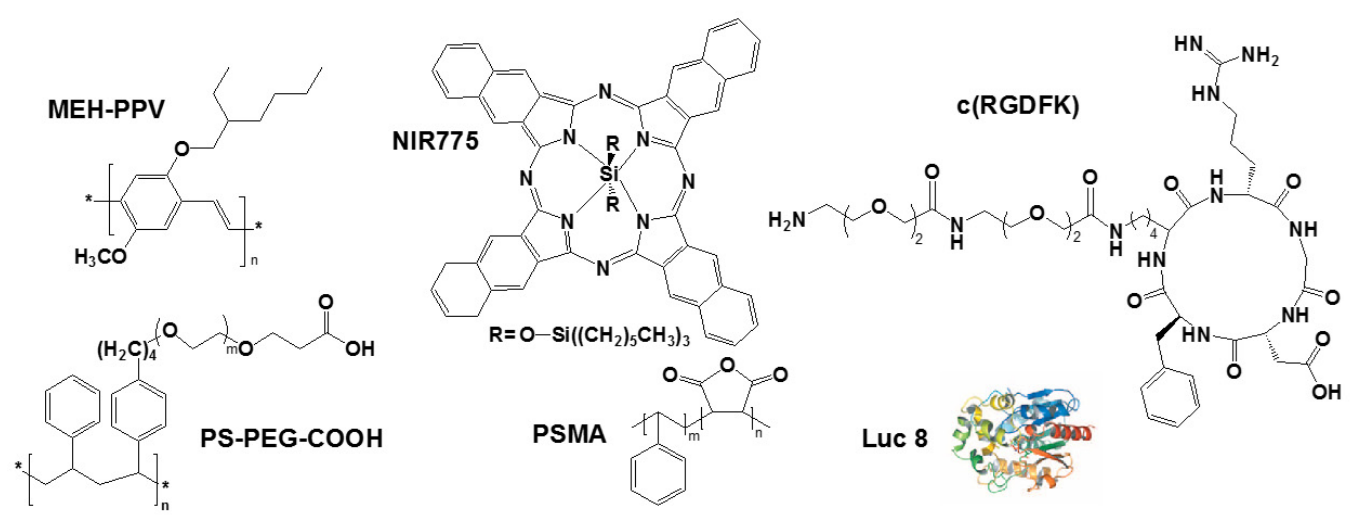

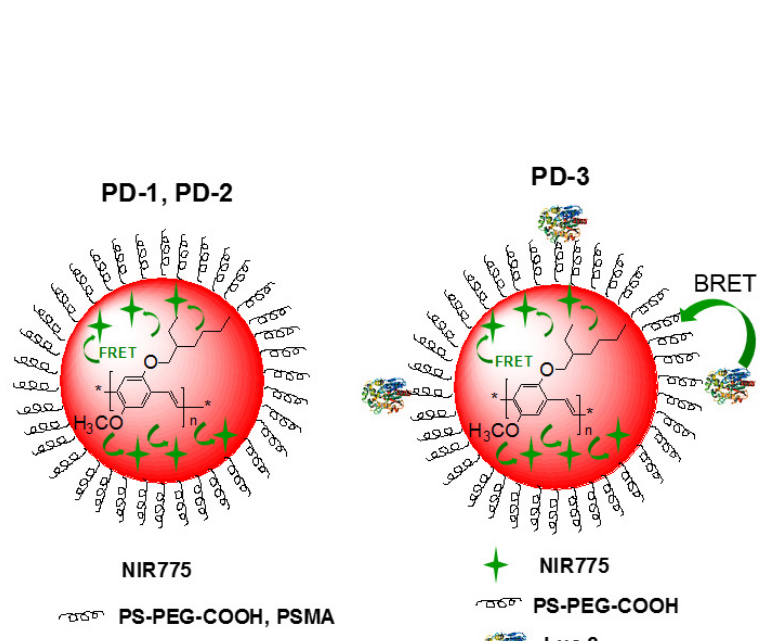

Suc 8
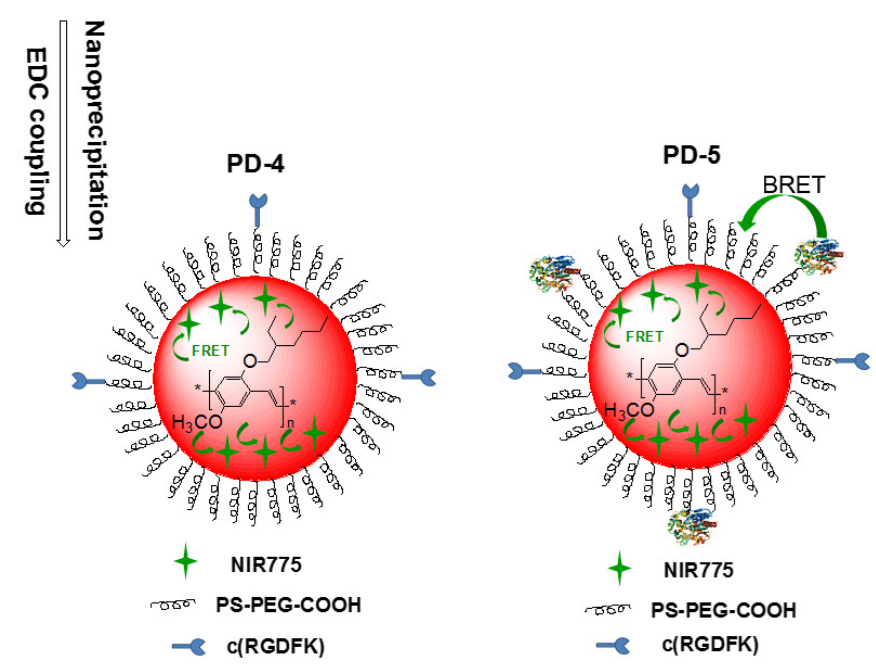

B

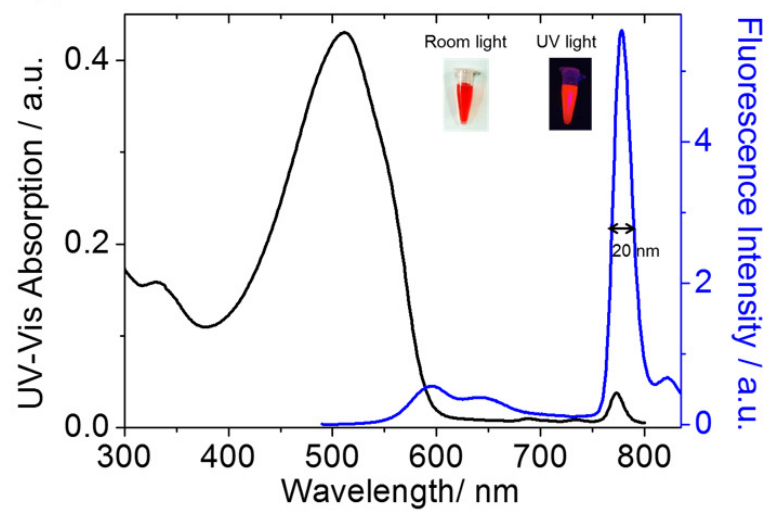

C

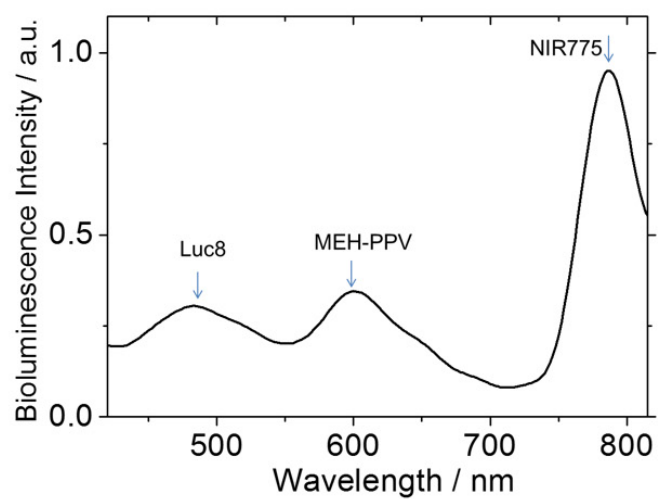

Scheme 1 (A) Chemical structures of MEH-PPV, polystyrene graft ethylene oxide functionalized with carboxyl groups (PS-PEG-COOH), poly(styrene-alt-maleic anhydride) (PSMA), NIR775, c(RGDFK) and Luc8, and the schematic depicts the preparation of the NIR polymer dots. PD-1:PS-PEG-COOH modified polymer dots; PD-2: PSMA modified polymer dots; PD-3:PS-PEG-COOH modified and Luc-8 conjugated polymer dots; PD-4: PS-PEG-COOH modified and RGD conjugated polymer dots; PD-5: PS-PEG-COOH modified, RGD and Luc-8 conjugated polymer dots. The biochemical energy generated from the Luc8-catalysed oxidation of coelenterazine transfers initially to the MEH-PPV polymer and is then relayed to doped NIR775 dye to produce NIR emission. Surface ligands, PS-PEG-COOH or PSMA, coat the nanoparticle to improve water solubility and biocompatibility. Tumor targeting ligands such as cyclic RGD peptides are conjugated to the polymer dots surface for in vivo cancer imaging. (B) UV-Vis absorption and fluorescence spectra of PD-1 polymer dots. Insets are the room light and UV light photographs of PD-1 polymer dots. The fluorescence photograph was obtained under $365 \mathrm{~nm}$ UV excitation, which showed red emission from the MEH-PPV. (C) Bioluminescence emission spectrum of PD-3 polymer dots in PBS buffer. ${ }^{[59-62]}$ 


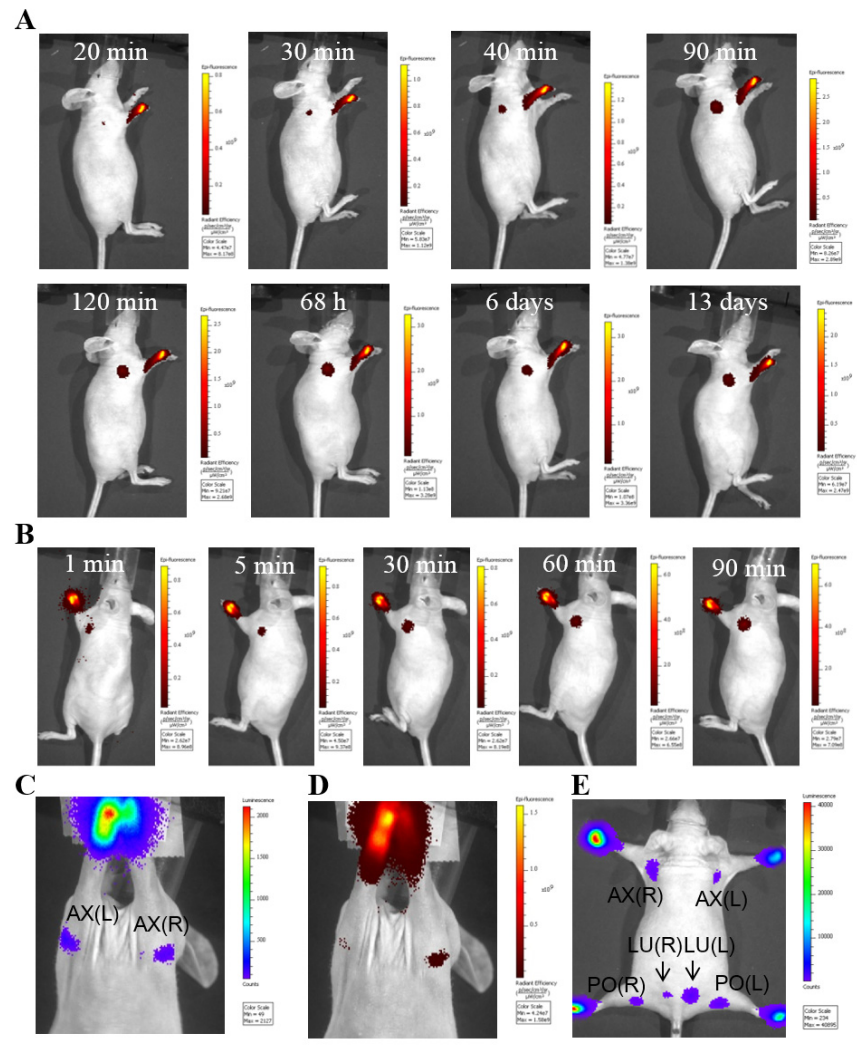

Figure 1 Fluorescence and bioluminescence imaging of lymphatic basins in mice by local injection. Time-dependent in vivo fluorescence imaging of axillary lymph node in the mice with injection of PD-2 $(10 \mu \mathrm{g})(\mathrm{A})$ and PD-1 (1 $\mu \mathrm{g})(\mathrm{B})$ intradermally in the forepaws. (C) Bioluminescence and (D) fluorescence imaging of lymphatic basins in a mouse 10 min after injection of PD-3 intradermally in the forepaws. (E) Bioluminescence imaging of lymphatic basins in a mouse with injection of PD-3 $(2 \mu \mathrm{g})$ intradermally in the forepaws. All bioluminescence images were acquired with $10 \mathrm{~s}$ exposure time; AX, axillary lymph node; LU, lumbar lymph node; PO, popliteal lymph node; L, left; R, right. ${ }^{[60]}$
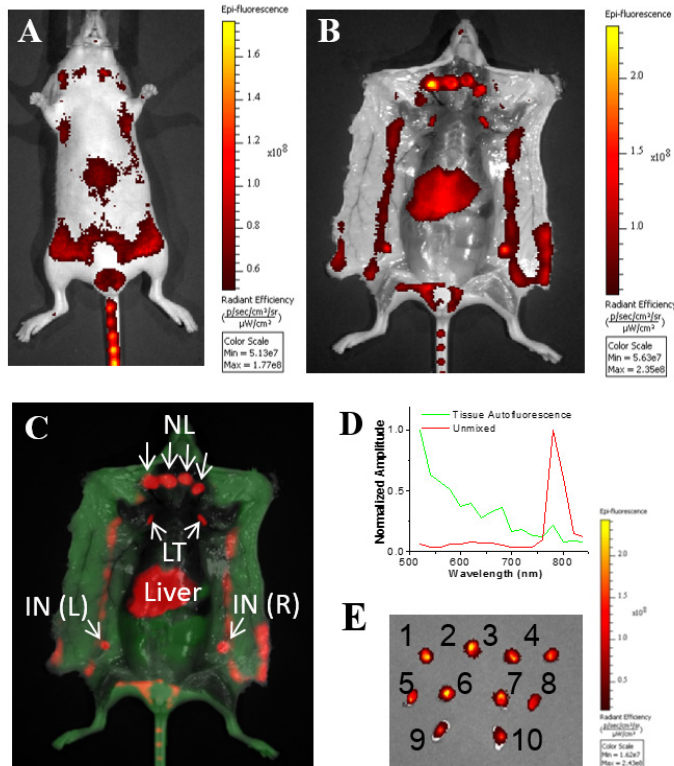

Figure 2 Fluorescence imaging of lymphatic basins in mice by tail-vein injection. (A-C) Fluorescence imaging of a mouse $24 \mathrm{~h}$ after the tail-vein injection of PD-1 (20 $\mu \mathrm{g})$. (B, C) Superficial skin was removed before imaging but peritoneum was left intact. (C) Autofluorescence is coded in green and PD-1 signal in red; AX, axillary lymph node; IN, inguinal lymph node; L, left; LT, lateral thoracic lymph node; NL, neck lymph nodes; R, right. (D) Fluorescence spectra of tissue autofluorescence (green) and the unmixed polymer dots signal (red) in the mouse. (E) Fluorescence image of lymph nodes excised from the mouse: 1—4, NL; 5, AX (left); 6, LT (left); 7, LT (right); 8, AX (right); 9, IN (left), 10, IN (right). ${ }^{[60]}$ 
dots reached $\sim 30 \mathrm{~nm}$. For in vivo tumor vasculature imaging, the NIR polymer dots PD-1 ( $25 \mu \mathrm{g}$ per mouse) prepared after 3,6 , and $9 \mathrm{~m}$ were injected intravenously into nude mice bearing a human glioblastoma U87MG tumor on the left shoulder (stomach position), respectively. $30 \mathrm{~min}$ post injection, strong NIR fluorescence signals were clearly observed in the U87MG tumor vasculature of the mice (Figure 3B). It was observed that the prepared NIR polymer dots were not uptaken by live U87MG cells in vitro, but accumulating in the U87MG tumor in vivo. ${ }^{[61]}$ It is probably because tumor tissue vasculatures are leaking and hyperpermeable allowing preferential accumulation of nanoparticles in the tumor vasculature and tumor interstitial space (called passive nanoparticle tumor targeting). In particular, no obvious fluorescence intensity change was detected among the three mice, indicating excellent long-term photostability of the NIR polymer dots for in vivo imaging.

A

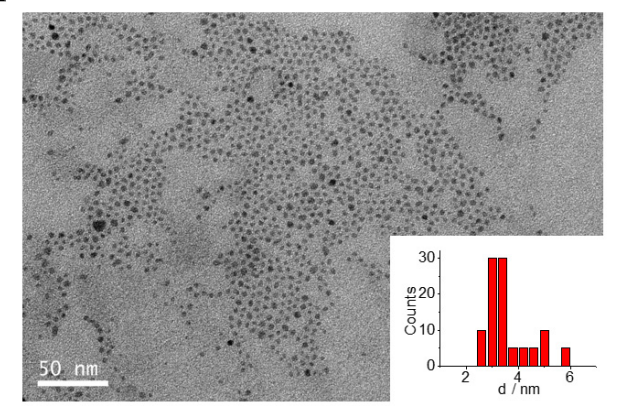

B

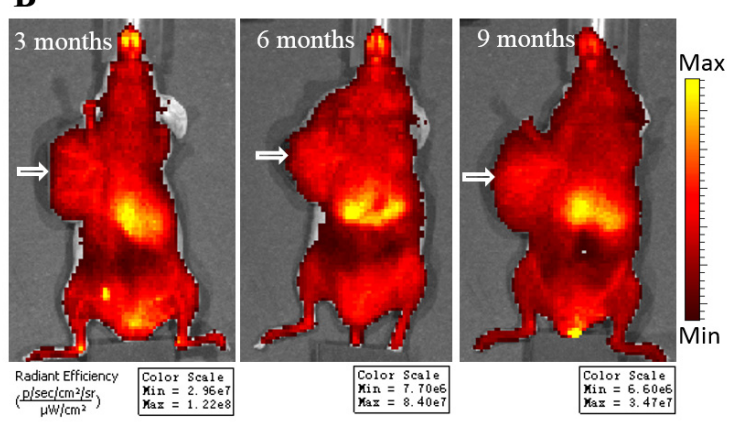

Figure 3 NIR polymer dots with ultra-small size were used for imaging tumor vasculature in vivo. (A) The TEM image of PD-1. Inset, the average diameter of NIR polymer dots obtained from TEM results. (B) In vivo fluorescence imaging of U87MG tumor bearing mice (tumors are indicated by white arrows) by the tail-vein injection of PD-1 prepared after 3, 6, and $9 \mathrm{~m}$, respectively. ${ }^{[61]}$

\section{Imaging of ultra-small tumors}

RGD peptides own a strong affinity for cell adhesion receptor integrin $\alpha_{v} \beta_{3}$, which plays a pivotal role in tumor angiogenesis, and have been used for in vivo imaging of a variety of cancers. Cyclic RGD peptides are conjugated to the surface of polymer dots for imaging human glioblastoma U87MG tumor xenografts in nude mice. $^{[60]}$
The NIR polymer dots PD-4 were injected intravenously into nude mice bearing a U87MG tumor $(\sim 8 \mathrm{~mm}$ in diameter) on the left shoulder, and the mice were imaged at multiple time points postinjection (Figure 4A). The in vivo fluorescence spectrum of the PD-4 was collected from 520 to $840 \mathrm{~nm}$ with a bandwidth of $20 \mathrm{~nm}$ and an excitation of $465 \mathrm{~nm}$. As early as 5 min postinjection, enhanced NIR fluorescence signals $(780 \mathrm{~nm})$ were observed in the U87MG tumor, and gradually increased over time. There were significant fluorescence signals from the skin after $1 \mathrm{~h}$, which complicated the detection of small size tumors.

The self-luminescing NIR polymer dots PD-5 can emit NIR light in the presence of the substrate of Luc8, coelenterazine, without external excitation. Therefore, they can provide further advantages in comparison with PD-4. After tail vein injection of PD-5, mice were imaged serially by bioluminescence imaging. At $5 \mathrm{~min}$ post injection, strong bioluminescence emission was observed in the U87MG tumor ( $\sim \mathrm{mm}$ in diameter) with little signal from other tissues. The bioluminescence signal ratio between the tumor and background was estimated from region of interest measurements to be above 100 at $2 \mathrm{~h}$ (Figure 4B). Furthermore, the efficiency of PD-5 in imaging smaller tumors (tumor size of $2-3 \mathrm{~mm}$ in diameter) (Figure $4 \mathrm{C}$ ) was tested. A strong bioluminescence signal was still observed in the U87MG tumor of the mice at $5 \mathrm{~min}, 2 \mathrm{~h}$ and $18 \mathrm{~h}$. The self-luminescing feature provided excellent tumor-tobackground ratio for imaging very small tumors.

A 5 min

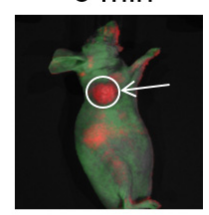

B

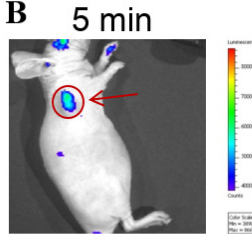

C 5 min

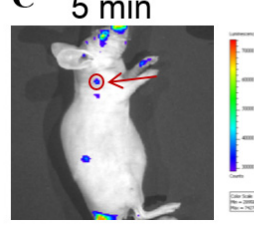

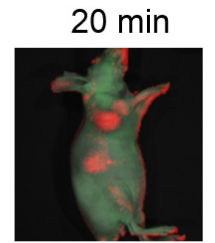

$2 \mathrm{~h}$

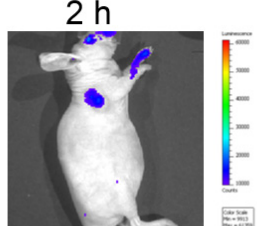

$2 \mathrm{~h}$

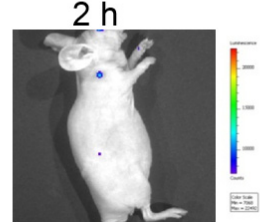

$1 \mathrm{~h}$

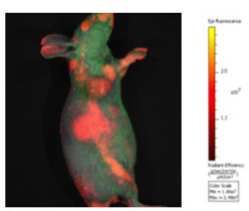

$24 \mathrm{~h}$

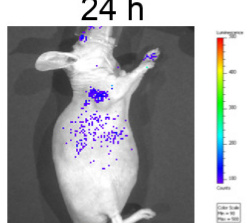

$18 \mathrm{~h}$

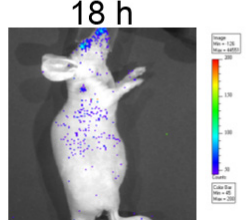

Figure 4 Time-dependent in vivo imaging of U87MG tumors in mice by the tail-vein injection of NIR polymer dots. (A) Fluorescence imaging of mouse with a tumor of $\sim 8 \mathrm{~mm}$ in diameter (indicated by a white arrow and circle) by the tail-vein injection of PD-4. Bioluminescence imaging of mice with small tumors of $\sim 5$ $\mathrm{mm}$ (B) and $\sim 2 \mathrm{~mm}$ (C) in diameter (indicated by red arrows and circles) by the tail-vein injection of PD-5. ${ }^{[60]}$ 


\section{Long-term tumor cell tracking}

The NIR775 dye-doped MEH-PPV polymer dots system was further used as fluorescent nanoprobes for in vitro $\mathrm{HeLa}$ cell labeling and in vivo long-term $\mathrm{HeLa}$ tumor tracking. ${ }^{[62]}$

Approximately $100 \%$ of $\mathrm{HeLa}$ cells were labeled with polymer dots PD-1 by direct cell labeling method under the fluorescence microscope. To investigate the long-term labeling capability and toxicity of the NIR polymer dots PD- $1,2 \times 10^{5} \mathrm{HeLa}$ cells treated with 20 $\mu \mathrm{g}$ of PD-1 were subcutaneously injected into a nude mouse. The mouse was imaged at different time after injection (Figure 5). The NIR polymer dots remain $75 \%$ of their fluorescence upon $7 \mathrm{~d}$ injection and still retains $28 \%$ of fluorescence after $23 \mathrm{~d}$, indicating the durable brightness and long-term photostability of the NIR polymer dots. Moreover, ex vivo imaging of tumors showed that strong NIR fluorescence signals were detected in the whole region of the tumor, suggesting that the NIR polymer dots could be inherited by daughter cells.
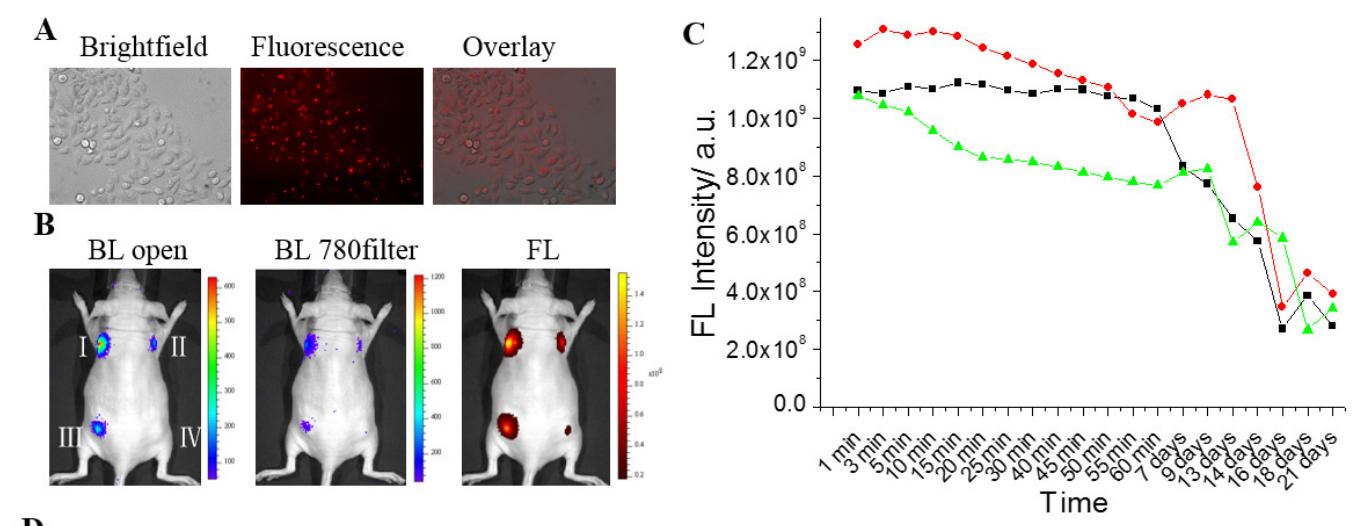

D
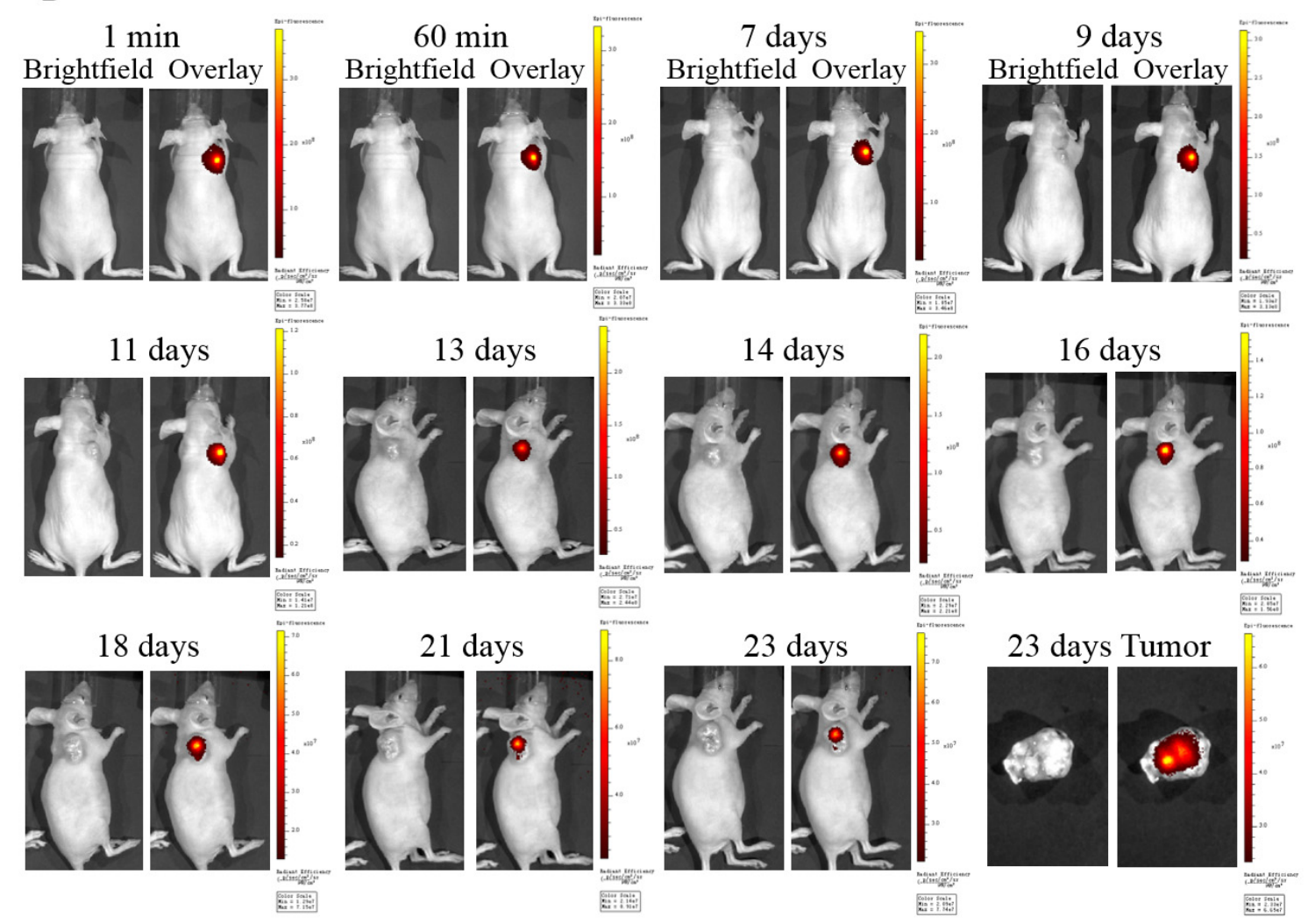

Figure 5 NIR polymer dots were used for the in vitro HeLa cell labeling and in vivo long-term HeLa tumor tracking. (A) Fluorescence imaging of HeLa cells labeled with PD-1. (B) In vivo bioluminescence (BL) and fluorescence (FL) imaging of the mouse with injection of different concentration of PD-3 subcutaneously (I: $3 \mu \mathrm{g}$, II: $0.3 \mu \mathrm{g}$, III: $0.6 \mu \mathrm{g}$, IV: $0.06 \mu \mathrm{g}$ ). (C) Region of interest (ROI) analysis of the fluorescence intensity of PD-1 labeled HeLa cells in the mice over time $(n=3)$. (D) Time-dependent in vivo brightfield and fluorescence imaging of the mouse with injection of PD-1 labeled HeLa cells and ex vivo fluorescence imaging of the tumor excreted from the mouse after 23 days injection. ${ }^{[62]}$ 
The difference in sensitivity between fluorescence and bioluminescence of NIR polymer dots PD-3 was also observed. The prepared Luc8-conjugated NIR polymer dots PD-3 with different concentration (3, 0.6, $0.3,0.06 \mu \mathrm{g}$ ) were subcutaneously injected into a nude mouse. The mouse was imaged immediately after an intravenous injection of $10 \mu \mathrm{g}$ of coelenterazine for bioluminescence imaging with $1 \mathrm{~min}$ acquisition time for no emission filter and $3 \mathrm{~min}$ acquisition time for NIR emission filter $(780 \pm 10 \mathrm{~nm})$. Following bioluminescence imaging, in vivo fluorescence imaging was carried out with $2 \mathrm{~s}$ acquisition time (excitation: $465 \pm 15 \mathrm{~nm}$; emission: $780 \pm 10 \mathrm{~nm}$ ) (Figure 5B). Intense NIR fluorescence signals were clearly visualized in the four injection sites, while bioluminescence imaging showed no obvious signal in the injection of $0.06 \mu \mathrm{g}$. Although bioluminescence imaging showed higher signals to background ratio, the NIR fluorescence imaging observed exhibited higher luminescence intensity even at low concentration. Compared to NIR bioluminescence imaging, NIR fluorescence imaging on the basis of PD-3 as a probe decreased the exposure time by $>$ 90 -fold, which benefitted the monitoring of quick interactions of cells.

\section{Conclusions and Outlook}

The NIR-emitting MEH-PPV polymer dots exhibited unique capabilities for in vivo imaging, such as good biocompatibility, durable brightness, excellent longterm photostability, and high sensitivity. The properties are crucial for imaging in living animals, but they do not exist in other optical imaging probes such as organic dyes and metallic nanoparticles (such as QDs). When properly designed, single polymer dots might be able to show simultaneously capabilities of multi-modal imaging (such as chemi/bioluminescence, photoacoustic, MRI) and therapy (such as photothermal and photodynamic therapies), which will provide unprecedented simplicity in designing a single nanoparticle-based multimodality image-guided therapy agent. Polymer dots thus represent a multifunctional nanoplatform that facilitates preclinical investigation into physiological and pathological processes in living subjects.

While polymer dots hold great promises for in vivo imaging, there are important issues that should be addressed before their advances towards potential clinical translations (for instance, the unspecific uptake in the skin and liver by the tail-vein injection). Strategies such as synthesis of a size smaller than the physiologic pore size of filtration slit in the glomerular capillary wall (5 $\mathrm{nm}$ ) are effective in reducing the uptake in the organs and being rapidly cleared out through urinary excretion. In addition, potential leakage of doped dyes from the polymer dot matrix was initially a concern. More synthetic strategies such as incorporating NIR dyes into polymer backbones and side chains, introducing moieties to induce intramolecular and intermolecular energy transfer, are effective in shifting the emission of polymer dots to the NIR region. Thereby, new chemistry for preparation of ultrasmall, biodegradable and high-performance NIR-emitting polymer dots is highly demanded for their advances towards potential clinical applications.

\section{Acknowledgement}

This work was supported by the Natural Science Foundation project of China (Nos. 81301261, 21374059, and 81671738), the Shanghai Pujiang Project (No. 13PJ1405000), and the Doctoral Fund of Ministry of Education of China (No. 20130073120098).

\section{References}

[1] Ntziachristos, V.; Ripoll, J.; Wang, L. H. V.; Weissleder, R. Nat. Biotechnol. 2005, 23, 313.

[2] McDonald, D. M.; Choyke, P. L. Nat. Med. 2003, 9, 713.

[3] Contag, C. H.; Bachmann, M. H. Annu. Rev. Biomed. Eng. 2002, 4, 235.

[4] Wagnieres, G. A.; Star, W. M.; Wilson, B. C. Photochem. Photobiol. 1998, 68, 603.

[5] van Dam, G. M.; Themelis, G.; Crane, L. M. A.; Harlaar, N. J.; Pleijhuis, R. G.; Kelder, W.; Sarantopoulos, A.; de Jong, J. S.; Arts, H. J. G.; van der Zee, A. G. J.; Bart, J.; Low, P. S.; Ntziachristos, V. Nat. Med. 2011, 17, 1315.

[6] Vahrmeijer, A. L.; Hutteman, M.; van der Vorst, J. R.; van de Velde, C. J.; Frangioni, J. V. Nat. Rev. Clin. Oncol. 2013, 10, 507.

[7] Wu, C. F.; Bull, B.; Szymanski, C.; Christensen, K.; McNeill, J. ACS Nano 2008, 2, 2415.

[8] Wu, C. F.; Schneider, T.; Zeigler, M.; Yu, J. B.; Schiro, P. G.; Burnham, D. R.; McNeill, J. D.; Chiu, D. T. J. Am. Chem. Soc. 2010, 132,15410 .

[9] Wei, L.; Zhou, P.; Yang, Q. X.; Yang, Q. Y.; Ma, M.; Chen, B.; Xiao, L. H. Nanoscale 2014, 6, 11351.

[10] Zhang, Y.; Yu, J.; Gallina, M. E.; Sun, W.; Rong, Y.; Chiu, D. T. Chem. Commun. 2013, 49, 8256.

[11] Wan, Y.; Zheng, L.; Sun, Y.; Zhang, D. J. Mater. Chem. B 2014, 2 , 4818.

[12] Wagh, A.; Qian, S. Y.; Law, B. Bioconjugate Chem. 2012, 23, 981.

[13] Pu, K. Y.; Shuhendler, A. J.; Valta, M. P.; Cui, L.; Saar, M.; Peehl, D. M.; Rao, J. H. Adv. Healthcare Mater. 2014, 3, 1292.

[14] Sun, W.; Hayden, S.; Jin, Y. H.; Rong, Y.; Yu, J. B.; Ye, F. M.; Chan, Y. H.; Zeigler, M.; Wu, C. F.; Chiu, D. T. Nanoscale 2012, 4, 7246.

[15] Feng, G.; Liu, J.; Genga, J.; Liu, B. J. Mater. Chem. B 2015, 3, 1135.

[16] Feng, G. X.; Li, K.; Liu, J.; Ding, D.; Liu, B. Small 2014, 10, 1212.

[17] Geng, J.; Li, K.; Pu, K.; Ding, D.; Liu, B. Small 2012, 8, 2421.

[18] Jin, Y.; Ye, F.; Zeigler, M.; Wu, C.; Chiu, D. T. ACS Nano 2011, 5, 1468.

[19] Pecher, J.; Mecking, S. Chem. Rev. 2010, 110, 6260.

[20] Zhu, C. L.; Liu, L. B.; Yang, Q.; Lv, F. T.; Wang, S. Chem. Rev. 2012 , $112,4687$.

[21] Pu, K.; Chattopadhyay, N.; Rao, J. J. Control Release 2016, 240, 312.

[22] Wu, C. F.; Chiu, D. T. Angew. Chem., Int. Ed. 2013, 52, 3086.

[23] Peng, H. S.; Chiu, D. T. Chem. Soc. Rev. 2015, 44, 4699.

[24] Li, K.; Liu, B. Chem. Soc. Rev. 2014, 43, 6570.

[25] Massey, M.; Wu, M.; Conroy, E. M.; Algar, W. R. Curr. Opin. Biotechnol. 2015, 34, 30.

[26] Pu, K.Y.; Liu, B. Adv. Funct. Mater. 2011, 21, 3408.

[27] Feng, L.; Zhu, C.; Yuan, H.; Liu, L.; Lv, F.; Wang, S. Chem. Soc. Rev. 2013, 42, 6620.

[28] Tian, Z. Y.; Yu, J. B.; Wu, C. F.; Szymanski, C.; McNeill, J. Nano- 
scale 2010, 2, 1999.

[29] Yuan, H. X.; Wang, B.; Lv, F. T.; Liu, L. B.; Wang, S. Adv. Mater. 2014, 26, 6978.

[30] Chan, Y. H.; Wu, P. J. Part. Part. Syst. Charact. 2015, 32, 11.

[31] Li, J.; Liu, J.; Wei, C. W.; Liu, B.; O'Donnell, M.; Gao, X. Phys. Chem. Chem. Phys. 2013, 15, 17006.

[32] Feng, G.; Ding, D.; Liu, B. Nanoscale 2012, 4, 6150.

[33] Xu, J.; Zhou, Y.; Cheng, G.; Liu, S.; Dong, M.; Huang, C. Luminescence 2015, 30, 451 .

[34] Botiz, I.; Freyberg, P.; Leordean, C.; Gabudean, A. M.; Astilean, S.; Yang, A. C.; Stingelin, N. ACS Appl. Mater. Interfaces 2014, 6, 4974.

[35] Köhler, A.; Hoffmann, S. T.; Bässler, H. J. Am. Chem. Soc. 2012, 134,11594

[36] Feist, F. A.; Zickler, M. F.; Basché, T. ChemPhysChem 2011, 12, 1499.

[37] Wang, W.; Alsmeier, J. H.; Schlaf, R. Langmuir 2013, 29, 6341.

[38] Hu, D. H.; Yu, J.; Barbara, P. F. J. Am. Chem. Soc. 1999, 121, 6936.

[39] Yu, Z. H.; Barbara, P. F. J. Phys. Chem. B 2004, 108, 11321

[40] Moscatelli, A.; Livingston, K.; So, W. Y.; Lee, S. J.; Scherf, U.; Wildeman. J.; Peteanu, L. A. J. Phys. Chem. B 2010, 114, 14430.

[41] Ding, D.; Liu, J.; Feng, G.; Li, K.; Hu, Y.; Liu, B. Small 2013, 9, 3093.

[42] Li, K.; Ding, D.; Huo, D.; Pu, K.; Thao, N. N. P.; Hu, Y.; Li, Z.; Liu, B. Adv. Funct. Mater. 2012, 22, 3107.

[43] Liu, J.; Geng, J.; Liu, B. Chem. Commun. 2013, 49, 1491

[44] Ding, D.; Li, K.; Qin, W.; Zhan, R.; Hu, Y.; Liu, J.; Tang, B. Z.; Liu, B. Adv. Healthcare Mater. 2013, 2, 500.

[45] Li, D. D.; Wang, J. X.; Ma, Y.; Qian, H. S.; Wang, D.; Wang, L.; Zhang, G.; Qiu, L.; Wang, Y. C.; Yang, X. Z. ACS Appl. Mater. Interfaces 2016, 8,19312.

[46] Yang, C.; Liu, H.; Zhang, Y.; Xu, Z.; Wang, X.; Cao, B.; Wang, M. Biomacromolecules 2016, 17, 1673.

[47] Seo, Y. H.; Singh, A.; Cho, H. J.; Kim, Y.; Heo, J.; Lim, C. K.; Park, S. Y.; Jang, W. D.; Kim, S. Biomaterials 2016, 84, 111.

[48] Lv, Y.; Liu, P.; Ding, H.; Wu, Y.; Yan, Y.; Liu, H.; Wang, X.;
Huang, F.; Zhao, Y.; Tian, Z. ACS Appl. Mater. Interfaces 2015, 7, 20640.

[49] Kim, S.; Lim, C. K.; Na, J.; Lee, Y. D.; Kim, K.; Choi, K.; Leary, J. F.; Kwon, I. C. Chem. Commun. 2010, 46, 1617.

[50] Hong, G.; Zou, Y.; Antaris, A. L.; Diao, S.; Wu, D.; Cheng, K.; Zhang, X.; Chen, C.; Liu, B.; He, Y.; Wu, J. Z.; Yuan, J.; Zhang, B.; Tao, Z.; Fukunaga, C.; Dai, H. Nat. Commun. 2014, 5, 4206.

[51] Yu, J. C.; Chen, Y.; L, Zhang, Y. Q.; Yao, X. K.; Qian, C. G.; Huang, J.; Zhu, S.; Jiang, X. Q.; Shen, Q. D.; Gu, Z. Chem. Commun. 2014, 50,4699

[52] Chen, C. P.; Huang, Y. C.; Liou, S. Y.; Wu, P. J.; Kuo, S. Y.; Chan, Y. H. ACS Appl. Mater. Interfaces 2014, 6, 21585.

[53] Liu, H. Y.; Wu, P. J.; Kuo, S.Y.; Chen, C. P.; Chang, E. H.; Wu, C. Y.; Chan, Y. H. J. Am. Chem. Soc. 2015, 137, 10420.

[54] Wu, I. C.; Yu, J.; Ye, F.; Rong, Y.; Gallina, M. E.; Fujimoto, B. S.; Zhang, Y.; Chan, Y. H.; Sun, W.; Zhou, X. H.; Wu, C.; Chiu, D. T. J. Am. Chem. Soc. 2015, 137, 173 .

[55] Wu, P. J.; Kuo, S. Y.; Huang, Y. C.; Chen, C. P.; Chan, Y. H. Anal. Chem. 2014, 86, 4831.

[56] Liou, S. Y.; Ke, C. S.; Chen, J. H.; Luo, Y. W.; Kuo, S. Y.; Chen, Y. H.; Fang, C. C.; Wu, C. Y.; Chiang, C. M.; Chan, Y. H. ACS Macro Lett. 2016, 5, 154.

[57] Zhu, H.; Fang, Y.; Zhen, X.; Wei, N.; Gao, Y.; Luo, K. Q.; Xu, C.; Duan, H.; Ding, D.; Chen, P.; Pu, K. Y. Chem. Sci. 2016, 7, 5118.

[58] Cao, F.; Xiong, L. Q. Chin. J. Chem. 2016, 34, 570.

[59] Palner, M.; Pu, K.; Shao, S.; Rao, J. Angew. Chem., Int. Ed. Engl. 2015, 54, 11477.

[60] Xiong, L. Q.; Shuhendler, A. J.; Rao, J. H. Nat. Commun. 2012, 3, 1193.

[61] Xiong, L. Q.; Cao, F. W.; Cao, X. M.; Guo, Y. X.; Zhang, Y. M.; Cai, X. Bioconjugate Chem. 2015, 26, 817 .

[62] Xiong, L. Q.; Guo, Y.; Zhang, Y.; Cao, F. J. Mater. Chem. B 2016, 4, 202. 